\title{
Influence of increased target dose inhomogeneity on margins for breathing motion compensation in conformal stereotactic body radiotherapy
}

\author{
Anne Richter*1, Kurt Baier ${ }^{1}$, Juergen Meyer ${ }^{2}$, Juergen Wilbert ${ }^{1}$, \\ Thomas Krieger ${ }^{1}$, Michael Flentje ${ }^{1}$ and Matthias Guckenberger ${ }^{1}$
}

\begin{abstract}
Address: ${ }^{1}$ Julius-Maximilians-University, Department of Radiation Oncology, Wuerzburg, Germany and ${ }^{2}$ University of Canterbury, Department of Physics and Astronomy, Christchurch, New Zealand

Email: Anne Richter* - richter_a3@klinik.uni-wuerzburg.de; Kurt Baier - baier_k@klinik.uni-wuerzburg.de; Juergen Meyer - juergen.meyer@canterbury.ac.nz; Juergen Wilbert - wilbert_j@klinik.uni-wuerzburg.de; Thomas Krieger - krieger_t@klinik.uniwuerzburg.de; Michael Flentje - flentje_m@klinik.uni-wuerzburg.de; Matthias Guckenberger - guckenberger_m@klinik.uni-wuerzburg.de

* Corresponding author
\end{abstract}

Published: 3 December 2008

BMC Medical Physics 2008, 8:5 doi:10.1186/1756-6649-8-5
Received: 28 May 2008

Accepted: 3 December 2008

This article is available from: http://www.biomedcentral.com/I756-6649/8/5

(C) 2008 Richter et al; licensee BioMed Central Ltd.

This is an Open Access article distributed under the terms of the Creative Commons Attribution License (http://creativecommons.org/licenses/by/2.0), which permits unrestricted use, distribution, and reproduction in any medium, provided the original work is properly cited.

\begin{abstract}
Background: Breathing motion should be considered for stereotactic body radiotherapy (SBRT) of lung tumors. Four-dimensional computer tomography (4D-CT) offers detailed information of tumor motion. The aim of this work is to evaluate the influence of inhomogeneous dose distributions in the presence of breathing induced target motion and to calculate margins for motion compensation.

Methods: Based on 4D-CT examinations, the probability density function of pulmonary tumors was generated for ten patients. The time-accumulated dose to the tumor was calculated using one-dimensional (ID) convolution simulations of a 'static' dose distribution and target probability density function (PDF). In analogy to stereotactic body radiotherapy (SBRT), different degrees of dose inhomogeneity were allowed in the target volume: minimum doses of $100 \%$ were prescribed to the edge of the target and maximum doses varied between $102 \%$ (PI02) and 150\% (PI 50). The dose loss due to breathing motion was quantified and margins were added until this loss was completely compensated.

Results: With the time-weighted mean tumor position as the isocentre, a close correlation with a quadratic relationship between the standard deviation of the PDF and the margin size was observed. Increased dose inhomogeneity in the target volume required smaller margins for motion compensation: margins of $2.5 \mathrm{~mm}, 2.4 \mathrm{~mm}$ and $1.3 \mathrm{~mm}$ were sufficient for compensation of $11.5 \mathrm{~mm}$ motion range and standard deviation of $3.9 \mathrm{~mm}$ in PI05, PI25 and PI50, respectively. This effect of smaller margins for increased dose inhomogeneity was observed for all patients. Optimal sparing of the organ-at-risk surrounding the target was achieved for dose prescriptions PI05 to PII8. The internal target volume concept over-compensated breathing motion with higher than planned doses to the target and increased doses to the surrounding normal tissue.

Conclusion: Treatment planning with inhomogeneous dose distributions in the target volume required smaller margins for compensation of breathing induced target motion with the consequence of lower doses to the surrounding organs-at-risk.
\end{abstract}




\section{Background}

The process of treatment planning and dose calculation in radiation therapy is currently based on a three-dimensional (3D) patient model. Temporal changes of the patients' anatomy due to breathing motion for e.g. lung tumors are not easily accounted for [1]. The use of standard, population based margins has been shown to be insufficient for a considerable proportion of the patients [2]. Different strategies have been developed for quantification of breathing motion [3-5] and for integration of the motion information into the planning and treatment workflow [6-12].

Non-gated treatment of the patient breathing freely without tumor tracking is the most straightforward technique: the requirements on the patients' pulmonary function and cooperation are low, the irradiation is performed continuously resulting in short total treatment times and no specific technical equipment is required. However, precise assessment of breathing induced target motion and calculation of adequate safety margins are essential for this technique.

Four-dimensional respiratory correlated computed tomography (4D-CT) is considered as the standard to gain detailed information about range and pattern of breathing induced organ motion $[13,14]$. The next step is the integration of this temporal dimension into the process of dose calculation and treatment planning. A straightforward approach to approximate the effect of tumor motion on the dose distribution is convolving a static dose distribution with a matrix containing the probability density function (PDF) of the moving organ [15-22].

Engelsman et. al [23] used this method to investigate the influence of breathing induced motion of pulmonary tumors and to calculate margins for compensation of this uncertainty. The margin was described by the displacement of the $95 \%$ isodose in the blurred dose profile compared to the static dose profile. That study was analogous to current clinical practice in fractionated radiotherapy, where the target volume is treated with a homogenous dose distribution.

However, for some clinical indications like cranial and extracranial stereotactic radiotherapy, inhomogeneous dose distributions are commonly used. In pulmonary stereotactic body radiotherapy (SBRT) the degree of dose inhomogeneity in the target volume varies widely: doses are prescribed to the $50 \%, 60 \%, 65 \%, 80 \%, 85 \%$ or $90 \%$ isodose $[24,25]$. Motion of the target in such inhomogeneous dose distributions is expected to yield different results compared to simulations based on a homogenous dose distribution.
It was the aim of the current work to simulate the effect of tumor motion in inhomogeneous dose distributions. Inhomogeneous dose distributions were generated by field size reduction without intensity modulation. The convolution model was used to simulate the motion of a target in a homogeneous medium and the motion simulation was focused on respiratory motion while setup errors were ignored. The decrease of treatment dose in the target due to motion was evaluated and margins for compensation of breathing motion were calculated. Different degrees of dose inhomogeneity in the target for a single beam profile were used. The results of this study are expected to be of clinical relevance especially for conformal stereotactic body radiotherapy of pulmonary and intra-hepatic lesions.

\section{Methods}

The investigations of this study are based on real patient data: ten consecutive patients (age $67 \pm 6$ years, female 3 , male 7) treated with SBRT for early stage non-small cell lung cancer or pulmonary metastases. Written informed consent was obtained from all patients.

For all ten patients a respiratory correlated 4D-CT study was acquired for treatment planning (Siemens Sensation Open; Siemens Medical Solutions, Erlangen, Germany). Details about 4D-CT image acquisition and image reconstruction have been described previously [24]. The motion range of the pulmonary target was evaluated after reconstruction of CT series in peak-inhalation and peakexhalation. The motion of the tumor in the predominant direction was chosen for one-dimensional (1D) simulations: this was the superior-inferior direction in all patients. A pressure sensor placed in an elastic belt around the patient's abdomen generated the external breathing signal (Anzai AZ-733V; Anzai Medical Solutions, Japan). The external breathing signal recorded during acquisition of the 4D-CT study was the basis for the calculation of the tumor PDF; the range of the PDF was equated with the maximum motion range of the tumor measured in the 4D-CT (Figure 1).

The patient specific parameters are listed in table 1; breathing motion was quantified by the range of the PDF $\left(\mathrm{A}_{\mathrm{Max}}\right)$, standard deviation (Eq. 1$)$ of the PDF $\left(\sigma_{\mathrm{PDF}}\right)$ and the time-weighted mean target position $\left(\mathrm{P}_{\text {Mean }}\right)$. The standard deviation of the motion signal $P$ was calculated using the position sample $\left(\mathrm{P}_{\mathrm{i}}\right)$, the mean target position $\left(\mathrm{P}_{\text {Mean }}\right)$ and the number of elements in the sample $(\mathrm{n})$.

$$
\sigma_{\mathrm{PDF}}=\sqrt{\frac{1}{n-1} \sum_{i=1}^{n}\left(P_{i}-P_{\text {Mean }}\right)^{2}}
$$



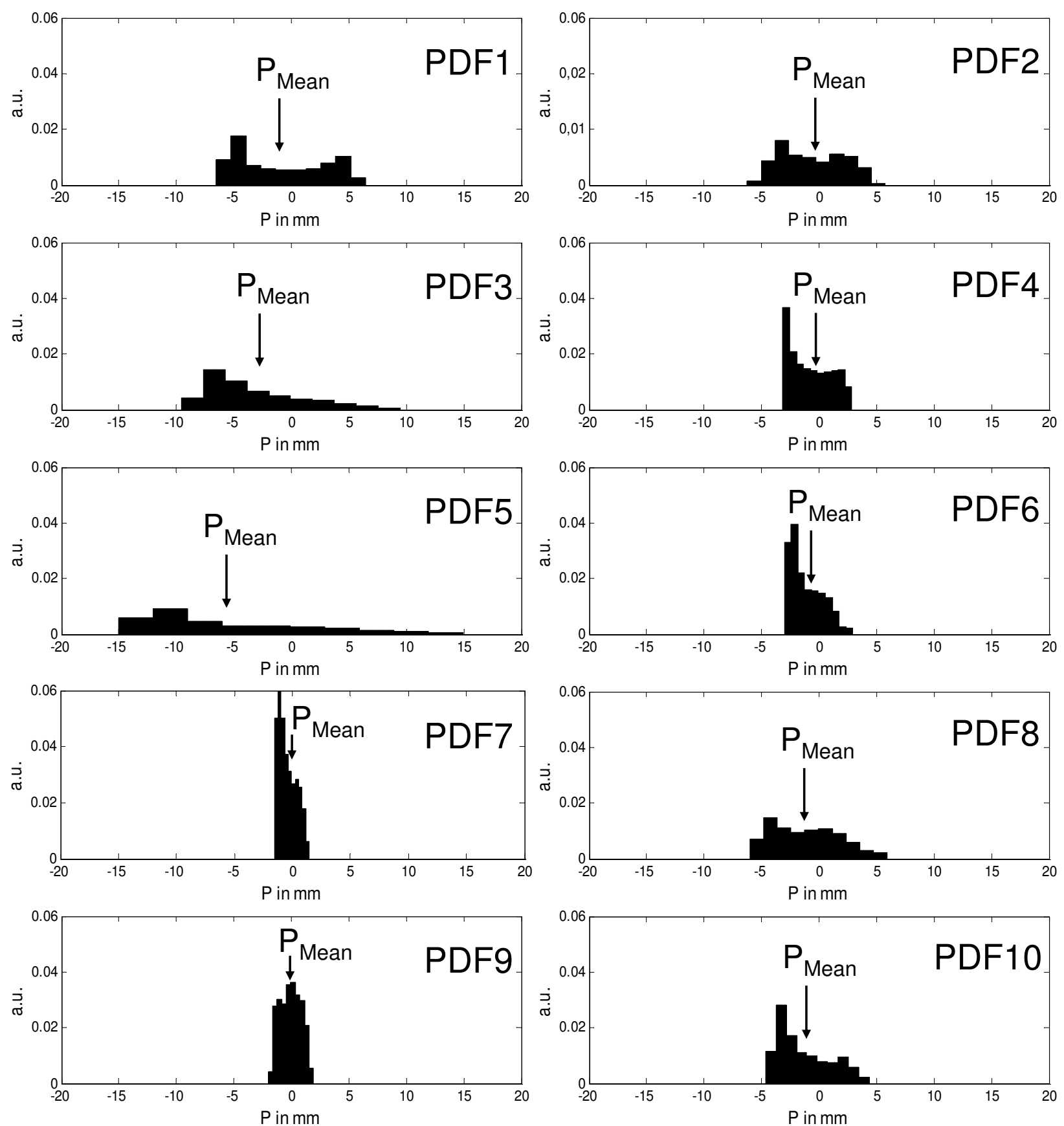

Figure I

Probability density functions of the pulmonary tumor for the 10 patients (exhalation and inhalation position have negative and positive numbers). The zero position is the geometrical centre of the PDF and the mean target position $\left(P_{\text {Mean }}\right)$ is marked with an arrow. 
Table I: Overview of patient data derived from 4D-CT imaging and the external breathing signal

\begin{tabular}{ccccc}
\hline Patient & $\begin{array}{c}\text { Tumor } \boldsymbol{\varnothing} \\
(\mathbf{m m})\end{array}$ & $\begin{array}{c}\mathbf{A}_{\mathbf{M a x}} \\
\mathbf{( m m})\end{array}$ & $\begin{array}{c}\sigma_{\mathbf{P D F}} \\
\mathbf{( m m})\end{array}$ & $\begin{array}{c}\mathbf{P}_{\text {Mean PDF }} \\
\mathbf{( m m})\end{array}$ \\
\hline $\mathbf{1}$ & 18 & 11.5 & 3.8 & -1.0 \\
$\mathbf{2}$ & 18 & 11.1 & 2.8 & -0.6 \\
$\mathbf{3}$ & 12 & 17.4 & 4.2 & -3.1 \\
$\mathbf{4}$ & 25 & 4.9 & 1.8 & -0.7 \\
$\mathbf{5}$ & 40 & 26.8 & 7.1 & -5.6 \\
$\mathbf{6}$ & 32 & 5.5 & 1.4 & -1.2 \\
$\mathbf{7}$ & 27 & 3.0 & 0.8 & -0.4 \\
$\mathbf{8}$ & 15 & 10.9 & 2.9 & -1.2 \\
$\mathbf{9}$ & 10 & 3.6 & 0.9 & -0.1 \\
$\mathbf{1 0}$ & 8 & 7.8 & 2.3 & -1.3 \\
\hline
\end{tabular}

Abbreviations: range of the probability density function (PDF) $A_{\text {Max }}$, standard deviation of the PDF $\sigma_{\mathrm{PDF}}$, time-weighted mean target position $\mathrm{P}_{\text {Mean }}$ : this position is defined as the distance from the geometrical centre of the PDF

A 1D convolution model was used to investigate the dosimetric influence of breathing induced tumor motion. The model was implemented in Matlab ${ }^{\mathrm{TM}}$ (The MathWorks, Inc., Natick, MA, USA) and allowed the variation of the following parameters: dose profile, field size, target size, motion range and shape of the PDF.

Dose profiles were generated by convolving an intensity profile with a pencil beam kernel while a homogeneous medium was assumed [26]. The intensity profile was a square profile simulating an open field shape. To ensure the correctness of the computed dose profiles, a comparison with measured profiles in a water phantom was performed with the following parameters: field size $24 \times 24$ $\mathrm{mm}$, depth $50 \mathrm{~mm}$, machine Elekta Synergy S equipped with the BeamModulator with $4 \mathrm{~mm}$ leaf width (Elekta Oncology Systems, Crawley, UK) and photon energy 6 $\mathrm{MV}$. The calculated and measured dose profiles were in good agreement with $0.07 \%$ mean error normalized to maximum value. The resolution of the simulated dose profiles was $0.1 \mathrm{~mm}$.

Assuming no breathing motion, a minimum dose of $100 \%$ was prescribed to the edge of the static target in the time-weighted average position $\mathrm{P}_{\text {Mean }}$. The centre of the target in this time-weighted average position was the isocentre as suggested by Wolthaus et al. [27]. According to the SBRT, different degrees of dose inhomogeneity in the target volume were simulated. The maximum dose in the tumor was varied between $102 \%$ and $150 \%$. The dose inhomogeneity was achieved by variation of the field size only, no step-and-shoot segments for intensity modulation were used. Figure 2 displays the prescription types P105, P125 and P150 while P105 corresponds to a plan with a minimum dose of $100 \%$ and a maximum dose of $105 \%$ within the target volume.

The time accumulated dose profile $D_{a c c}$ was calculated by convolving the static reference dose profile $D_{0}(x)$ with the variation kernel $P D F(x)$.

$$
D_{a c c}=\int D_{0}\left(x-x^{\prime}\right) \bullet P D F(x) d x^{\prime}
$$

This resulted in a blurring of the dose gradient and consequently reduced the accumulated dose to the target volume (Figure 3a). For quantification of the motion effect, the dose to the static target was compared with the dose after motion simulation. The minimum dose to the target $\left(D_{\text {Min }}\right)$ was considered as the treatment dose. Then, field sizes were symmetrically enlarged in steps of $0.1 \mathrm{~mm}$ until the dose reduction due to breathing motion was completely compensated (Figure $3 \mathrm{~b}$ ). The position of the dose profile was shifted and optimized until the tumor received equal doses to the edges. A margin was defined as the increase in field size in one direction; the total increase in field size is consequently twice the one-sided margin. This target volume concept for compensation of breathing motion was termed as "optimization of the surrounding isodose" (OSI concept). Additional blurring of the dose profile due to setup errors were not considered in this work.

Additionally, the influence of motion on the dose to the region surrounding the tumor (organs-at-risk lung or liver) was investigated. This region corresponds to the pulmonary/hepatic tissue superior and inferior to the target in a 1D patient model, because target motion in superiorinferior direction was modelled. In real patient treatment, a significant dose is delivered to healthy tissue in the entrance and exit regions of the beam (axial direction) assuming a predominant coplanar beam set-up. This is not considered in a 1D model. Consequently, doses to the surrounding organ-at-risk were transformed into a 2D model in a sagittal plane: with a coplanar opposing field design, the dose distribution in superior-inferior direction was extrapolated in anterior-posterior direction. Doses in a 2D sagittal plane with extensions of $15 \mathrm{~cm}$ in superiorinferior and $12 \mathrm{~cm}$ anterior-posterior direction were calculated, respectively; the dose inside the target (equal tumor size in superior-inferior and anterior-posterior direction) was excluded for dose calculation to this organ-at-risk. As the mean lung dose was shown to be predictive for radiation induced pulmonary toxicity [28], the mean dose in this region was calculated $\left(D_{\text {Mean Surr }}\right)$.

The simulation in this study was divided into four parts to analyse the influence of the parameters motion range, dose inhomogeneity and motion pattern, separately. 


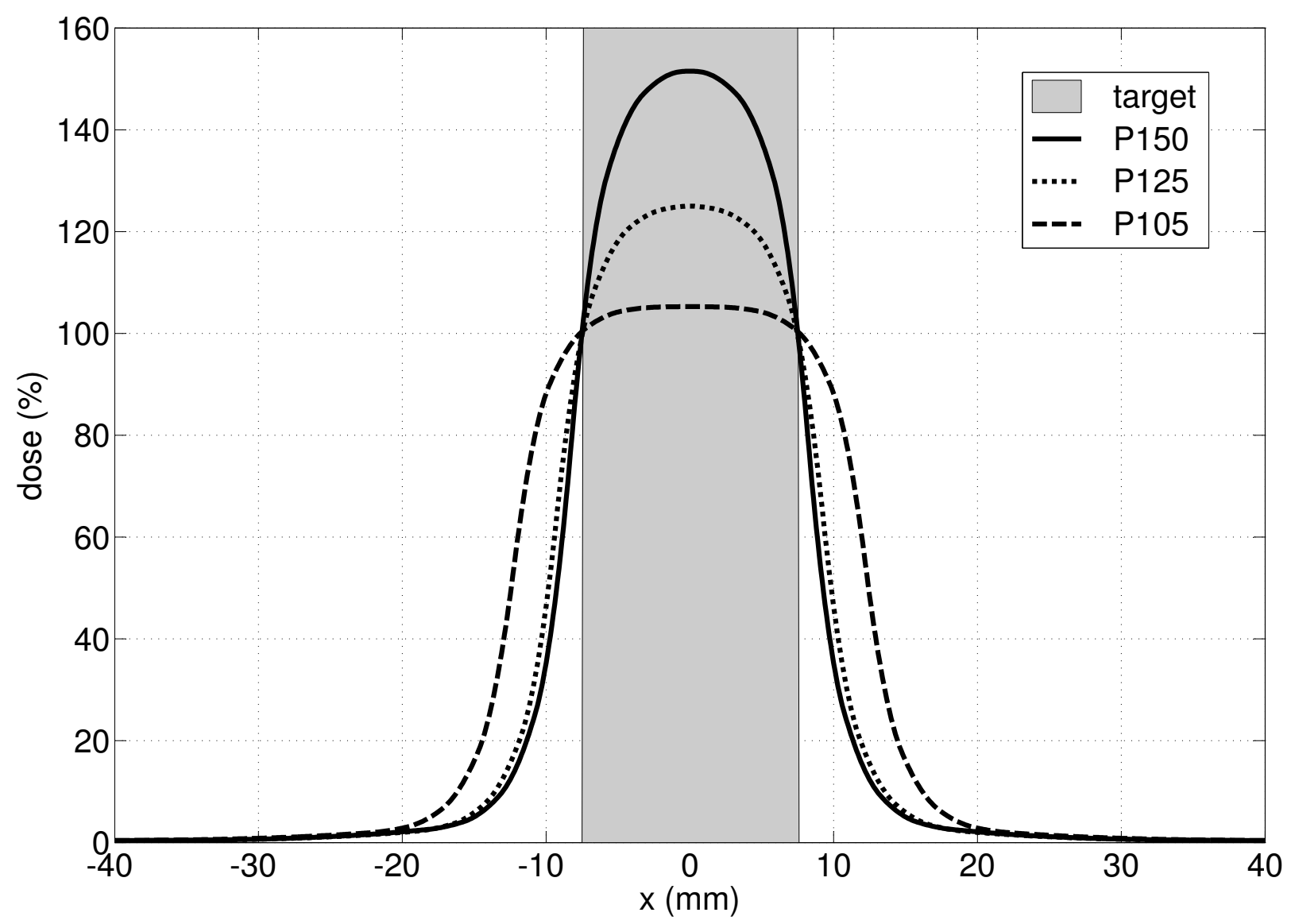

Figure 2

Illustration of three different dose prescriptions PI05, PI25 and PI50. The minimum dose was set to $100 \%$ at the edges of the target with maximum doses of $105 \%$, $125 \%$ and $150 \%$.

\section{Range of breathing motion}

The first part of the study systematically quantified the influence of the motion range on the dose to target. For a single breathing pattern (PDF\#1) and a target size of 20 $\mathrm{mm}$, the motion range $A_{\mathrm{Max}}$ was varied ranging from 2 $\mathrm{mm}$ to $30 \mathrm{~mm}$. These simulations were performed based on dose prescriptions P105, P125 and P150, which are most frequently used in pulmonary and hepatic SBRT. Margins necessary for compensation of the dose loss due to breathing motion were calculated.

\section{Breathing pattern}

The second part of the study systematically evaluated the influence of the breathing pattern. For one single target size of $20 \mathrm{~mm}$ and $\mathrm{A}_{\mathrm{Max}}$ of $15 \mathrm{~mm}$ the breathing pattern was varied with the ten clinically observed PDFs. The simulation was performed for P105, P125 and P150 and margins for motion compensation were calculated.

\section{Dose inhomogeneity}

The third part of the study systematically evaluated the influence of the dose inhomogeneity in the target on margins necessary for compensation of breathing motion. Dose prescriptions from P102 to P150 were simulated. This was done exemplary for three different patients (\#1, $\# 5, \# 8$ ) and the corresponding tumor PDF, $A_{M a x}$ and tumor size. Simulations for P105, P125 and P150 were performed using all ten patient data (Table 1). Additionally, the influence of dose inhomogeneity on $\mathrm{D}_{\text {Mean Surr }}$ the mean dose to the tumor surrounding region, was evaluated.

\section{ITV versus OSI target volume concept}

The internal target volume (ITV) concept was evaluated as this is most commonly used in SBRT treatment: the ITV encompassed the target during the whole breathing cycle and the $100 \%$ dose was prescribed to cover the ITV. 

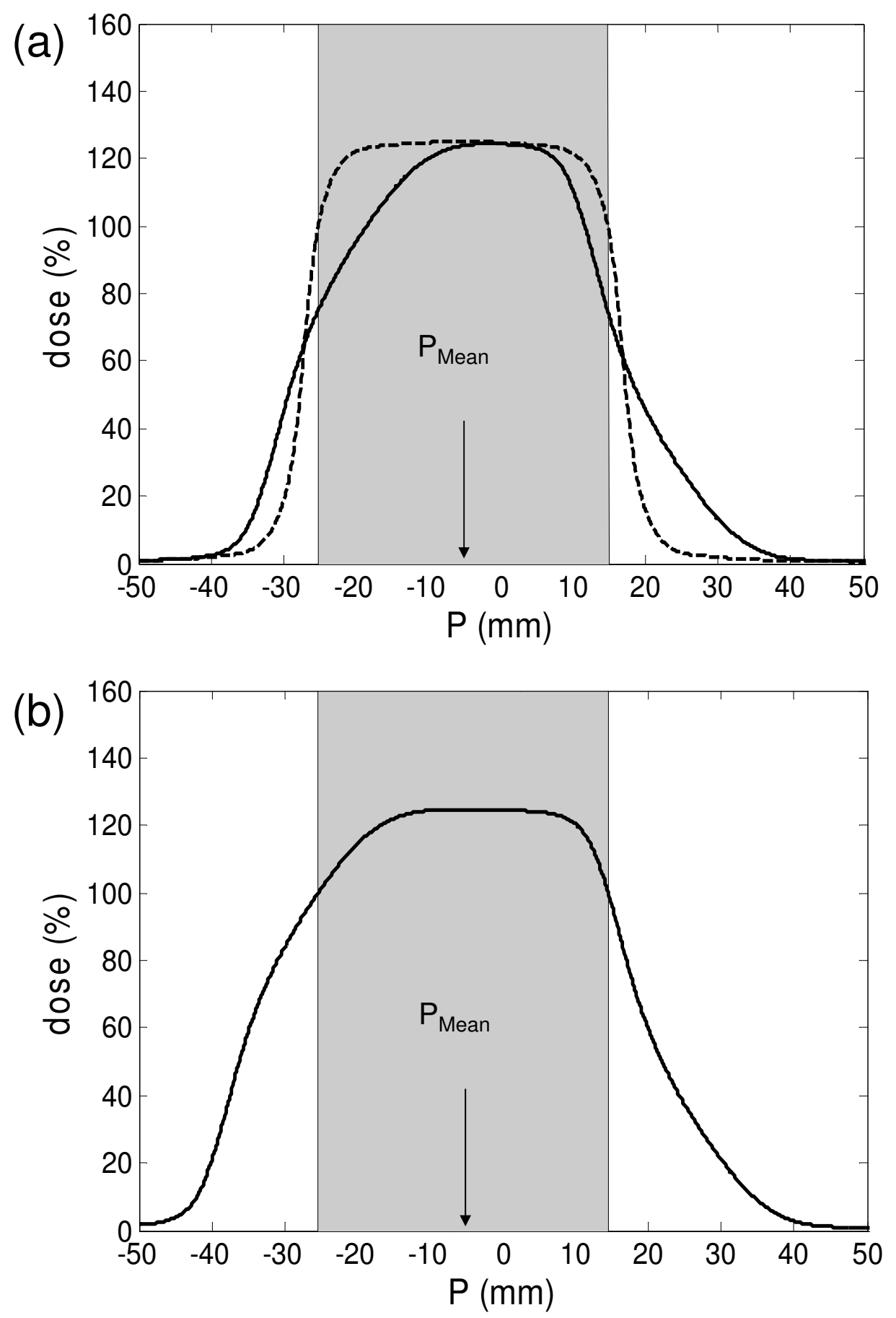

Figure 3

Workflow of convolution simulation for patient \#5 for prescription type PI 25 (the shaded area represents the target in the time weighted mean position $\mathbf{5 . 6} \mathbf{~ m m}$ ). (a) The dose to the target without (dashed line) and with consideration of motion (solid line). (b) The accumulated dose with an additional margin of $4.9 \mathrm{~mm}$ for motion compensation. 
Results of the OSI and ITV concepts were compared with dose prescriptions of P105, P125 and P150.

\section{Results}

Range of breathing motion

The first simulation evaluated the influence of the motion range $A_{M a x}$ on the dose to the target. An increasing range of breathing motion resulted in lower $\mathrm{D}_{\mathrm{Min}}$ to the target. For motion compensation, the optimal isocentre position for equal doses at both edges of the target volume agreed closely with the time-weighted mean target position for 8 of 10 patients. Differences of 0.9 and $2.1 \mathrm{~mm}$ were observed for patients 3 and 5 . These patients showed large motion amplitudes of 17 and $27 \mathrm{~mm}$ with highly asymmetrical breathing patterns.

A polynomial relationship of second order was found between $\sigma_{\mathrm{PDF}}$ and the margin size: for target motion between $2 \mathrm{~mm}$ and $30 \mathrm{~mm}$ the margin increased quadrat- ically (coefficients of determination (COD) 0.99). For P105, a margin of $2 \mathrm{~mm}$ was needed for $A_{M a x}=10 \mathrm{~mm}$ and $\sigma_{\mathrm{PDF}}=3.4 \mathrm{~mm}$. If the motion $\left(\mathrm{A}_{\mathrm{Max}}\right.$ and $\left.\sigma_{\mathrm{PDF}}\right)$ was twice as large, the margin size increased to $6 \mathrm{~mm}$. Results for the three different prescriptions are shown in Figure 4. A higher degree of dose inhomogeneity resulted in smaller margins for motion compensation. For example, to compensate the dose loss due to target motion of $\sigma_{\mathrm{PDF}}=6.8$ $\mathrm{mm}$ and $\mathrm{A}_{\mathrm{Max}}=20 \mathrm{~mm}$, margins of $6 \mathrm{~mm}, 5.5 \mathrm{~mm}$ and 2.8 $\mathrm{mm}$ were necessary for P105, P125 and P150, respectively. Isodose levels close to the $50 \%$ ideally don't move at all under dose blurring. In Figure 3 it was demonstrated, that especially smaller isodose (e.g. 65\%) levels of the accumulated dose profile were shifted less than higher isodose levels $(100 \%)$.

\section{Breathing pattern}

The influence of different breathing patterns on the margin size was investigated while $\mathrm{A}_{\mathrm{Max}}$ was maintained con-

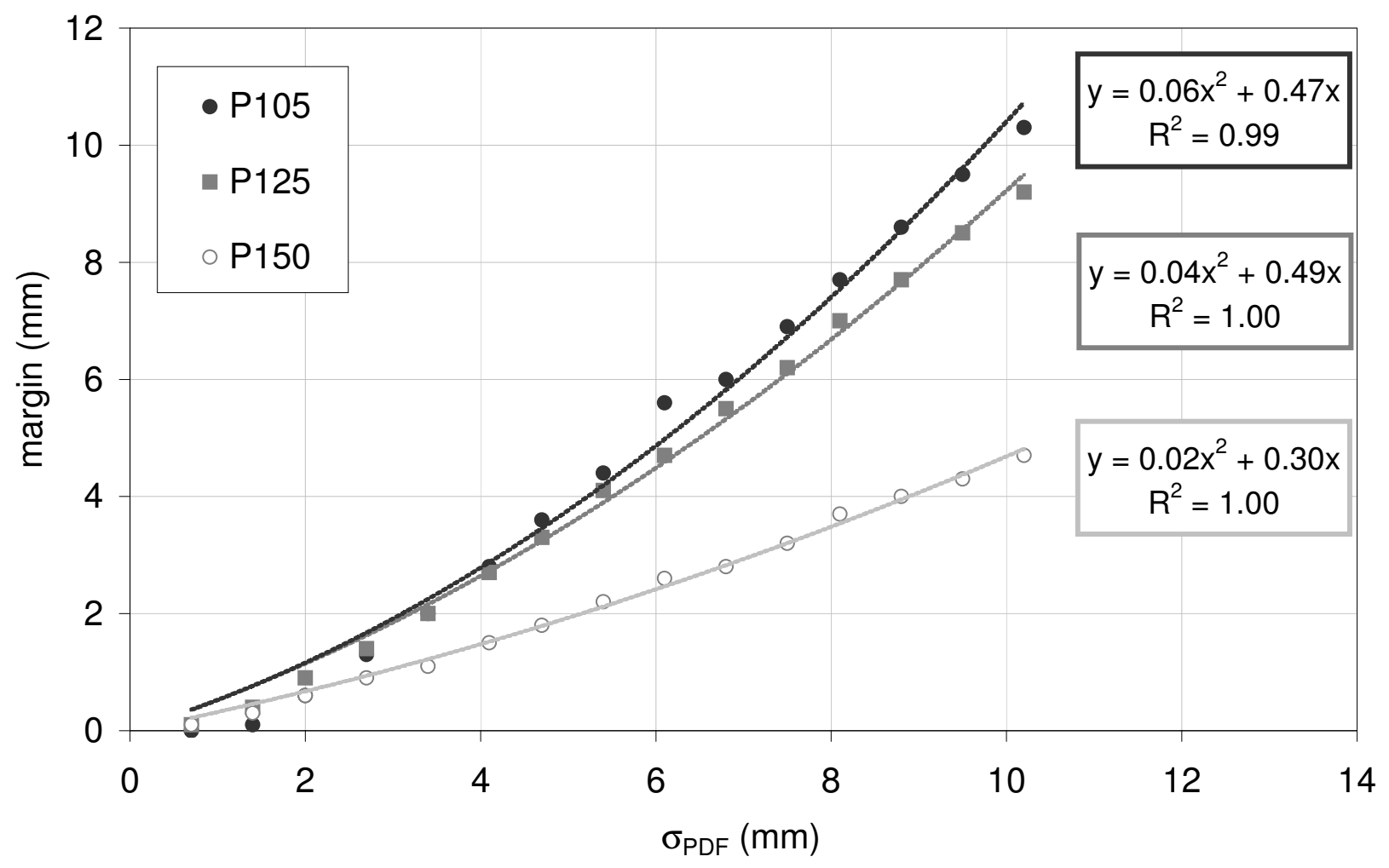

Figure 4

Margins for compensation of breathing induced dose loss to the target as a function of target motion ( $\left.\sigma_{\mathrm{PDF}}\right)$ for PDF\#I. 
stant at $15 \mathrm{~mm}$ : due to differences in the shapes of the PDFs, the $\sigma_{\mathrm{PDF}}$ ranged between $3.8 \mathrm{~mm}$ and $5 \mathrm{~mm}$. For the three prescription types (P105, P125 and P150), the data showed larger margins for increased $\sigma_{\mathrm{PDF}}$. Despite a constant $\mathrm{A}_{\mathrm{Max}^{\prime}}$ the margins for motion compensation varied between $2.7 \mathrm{~mm}$ and $4.1 \mathrm{~mm}$ for P105. Again, larger margins were needed for P105 than for P150 (Figure 5). These results show that $\sigma_{\mathrm{PDF}}$ is a more robust parameter to describe the target motion and PDF shape than $A_{\max }$ which characterizes only the width of target motion.

\section{Dose inhomogeneity}

The third simulation evaluated the influence of dose inhomogeneity in the target on margins for compensation of breathing motion. The dose prescription was varied from P102 to P150, margins were increased until the minimum dose to the target was $100 \%$. The results are illustrated in Figure 6. Margins were smallest for P150 and largest for P109 and P105: for $\mathrm{A}_{\mathrm{Max}}$ of $11.5 \mathrm{~mm}$ and target size of $18 \mathrm{~mm}$ (patient \#1), margins of $1.3 \mathrm{~mm}, 2.4 \mathrm{~mm}$ and $2.5 \mathrm{~mm}$ were required for P150, P125 and P105, respectively. The advantage of smaller margins for increased target dose inhomogeneity was observed for all three patients but the benefit was more obvious for large tumor motion.

The investigation of different degrees of dose inhomogeneity in the target showed generally an increase in margin size for more homogeneous target dose. Depending on the shape of the PDF, we observed a small decrease in margin size from P105 or P109 to P102 (Figure 6). The decrease was caused by convolving a gaussian shaped profile with a non symmetric PDF. Before convolution, the curvature of the static profile was monotonically decreasing from 66\% (P150) to 98\% (P102) isodose level. This was not the case for the accumulated dose profile which was deformed depending on the shape of the PDF. The distance of the prescription isodose level between static

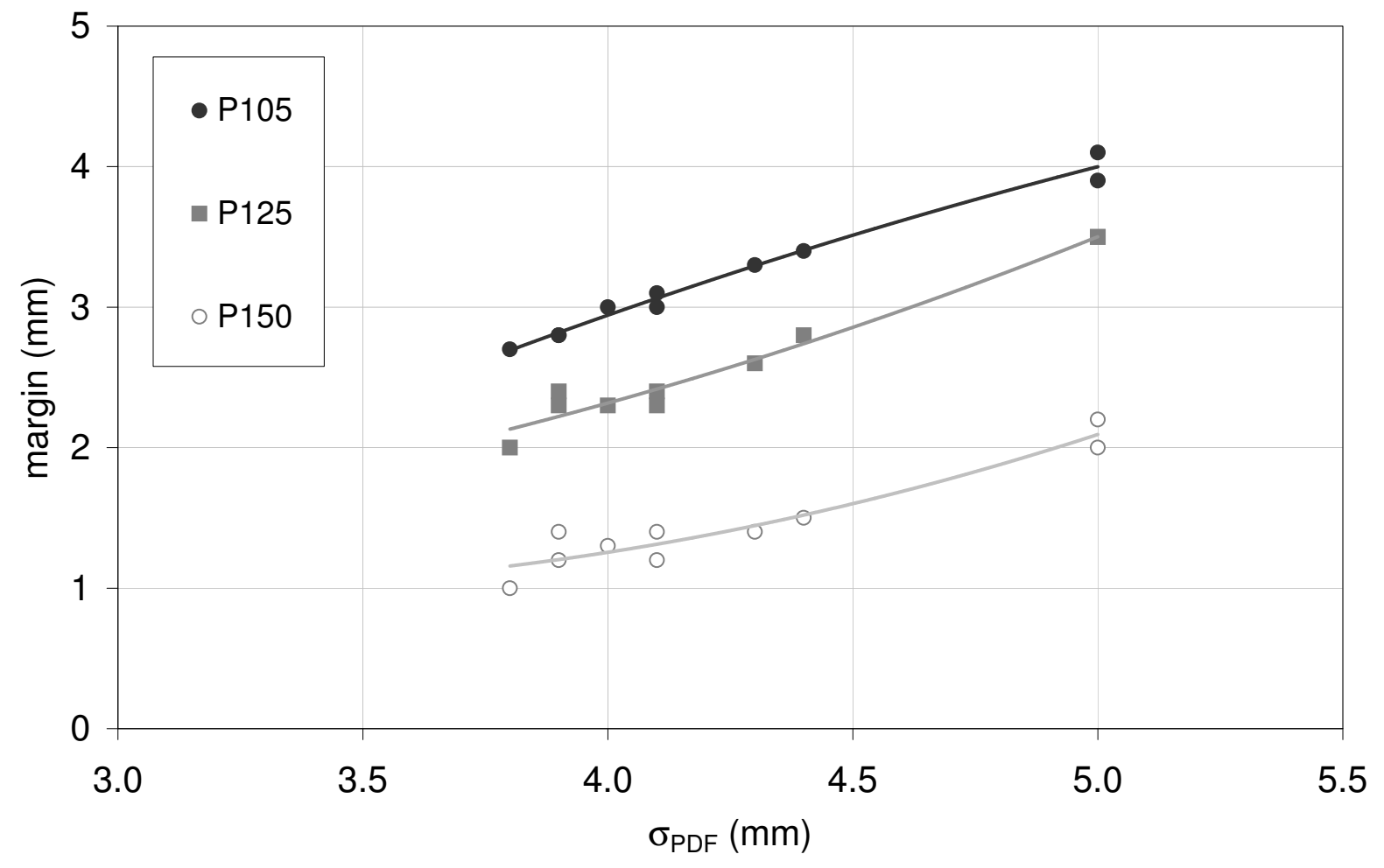

\section{Figure 5}

Margins size as a function of the standard deviation of target motion $\left(\sigma_{\mathrm{PDF}}\right)$ for the ten clinically observed breathing patterns; motion range $(15 \mathrm{~mm})$ and target size $(20 \mathrm{~mm})$ remained constant in this experiment. 


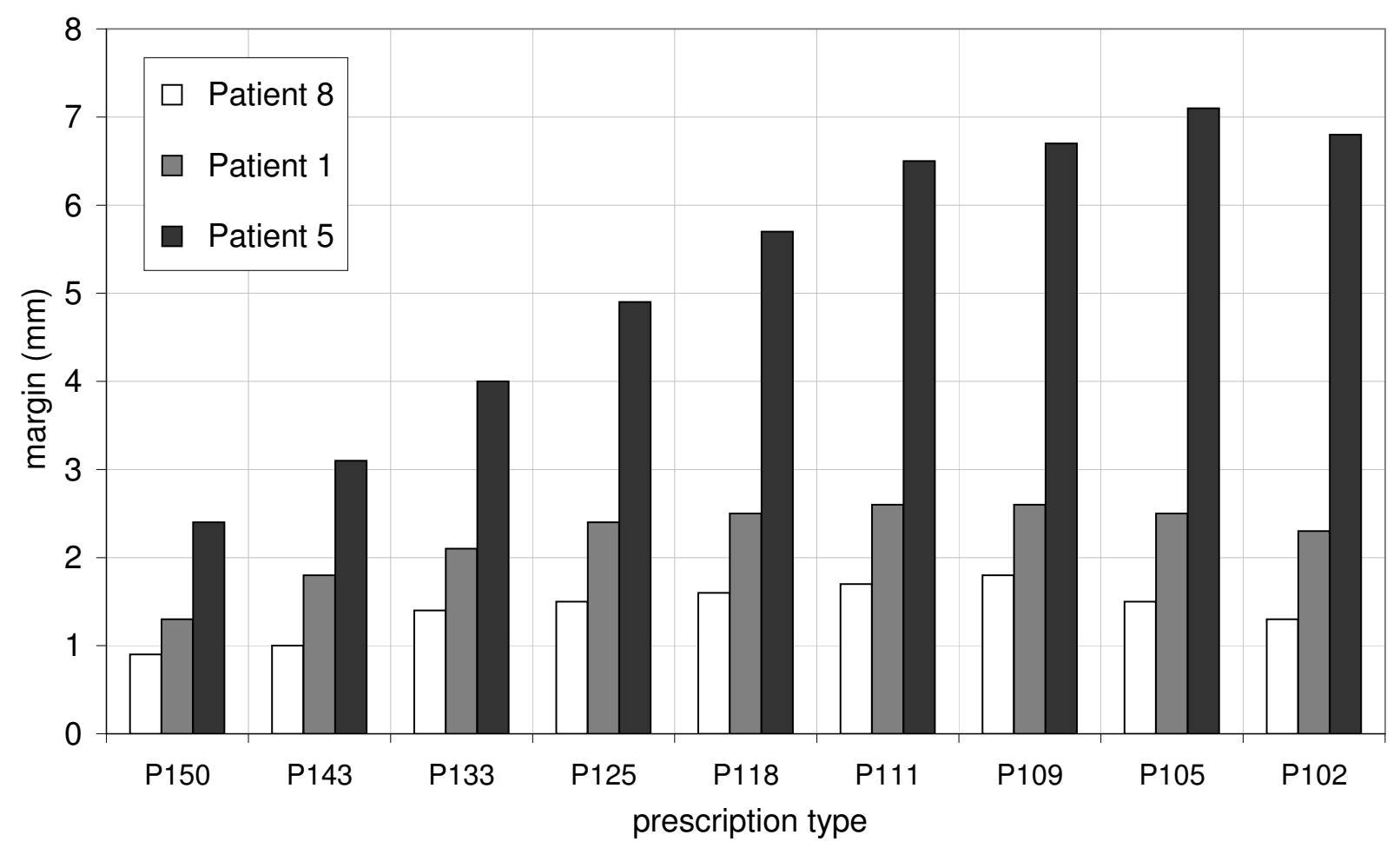

Figure 6

Margins for compensation of breathing induced dose loss to the target as a function of the dose prescription for three particular patients (\#8, \#I, \#5).

and accumulated profile was largest for P105 and P109 which resulted in the maximum compensation margins. The distance decreased for larger prescription isodose levels and therefore the necessary margin size was decreasing as well.

Figure 7 shows the mean dose to the organ-at-risk surrounding the target as a function of dose inhomogeneity from P150 to P102 for three patients. Motion compensation with dose inhomogeneities from P105 to P118 resulted in the lowest mean dose to surrounding organ-atrisk. Despite smaller safety margins in superior-inferior direction for larger dose inhomogeneity than P118, the mean dose to the surrounding organ-at-risk increased due to higher doses in the entrance and exit region of the radiation beams.
The effect of smaller safety margins with increased dose inhomogeneity was validated for all ten patients (Figure 8).

\section{ITV versus OSI target volume concept}

The comparison of motion compensation using the ITV concept and the OSI concept showed differences for margins, target dose and $\mathrm{D}_{\text {Mean Surr }}$ (Table 2). Margins for compensation of breathing motion were smaller based on the OSI concept compared to the ITV concept. As a consequence, the ITV concept over-compensated the effects of breathing motion with higher than planned doses to the target: the ITV concept increased $\mathrm{D}_{\mathrm{Min}}$ to the target by 3.6 $\pm 0.7 \%, 17.8 \pm 4.0 \%, 37.2 \pm 8.9 \%$ for P105, P125 and P150, respectively. Simultaneously, $D_{\text {Mean Surr was }}$ increased for the ITV concept compared to the OSI concept. 


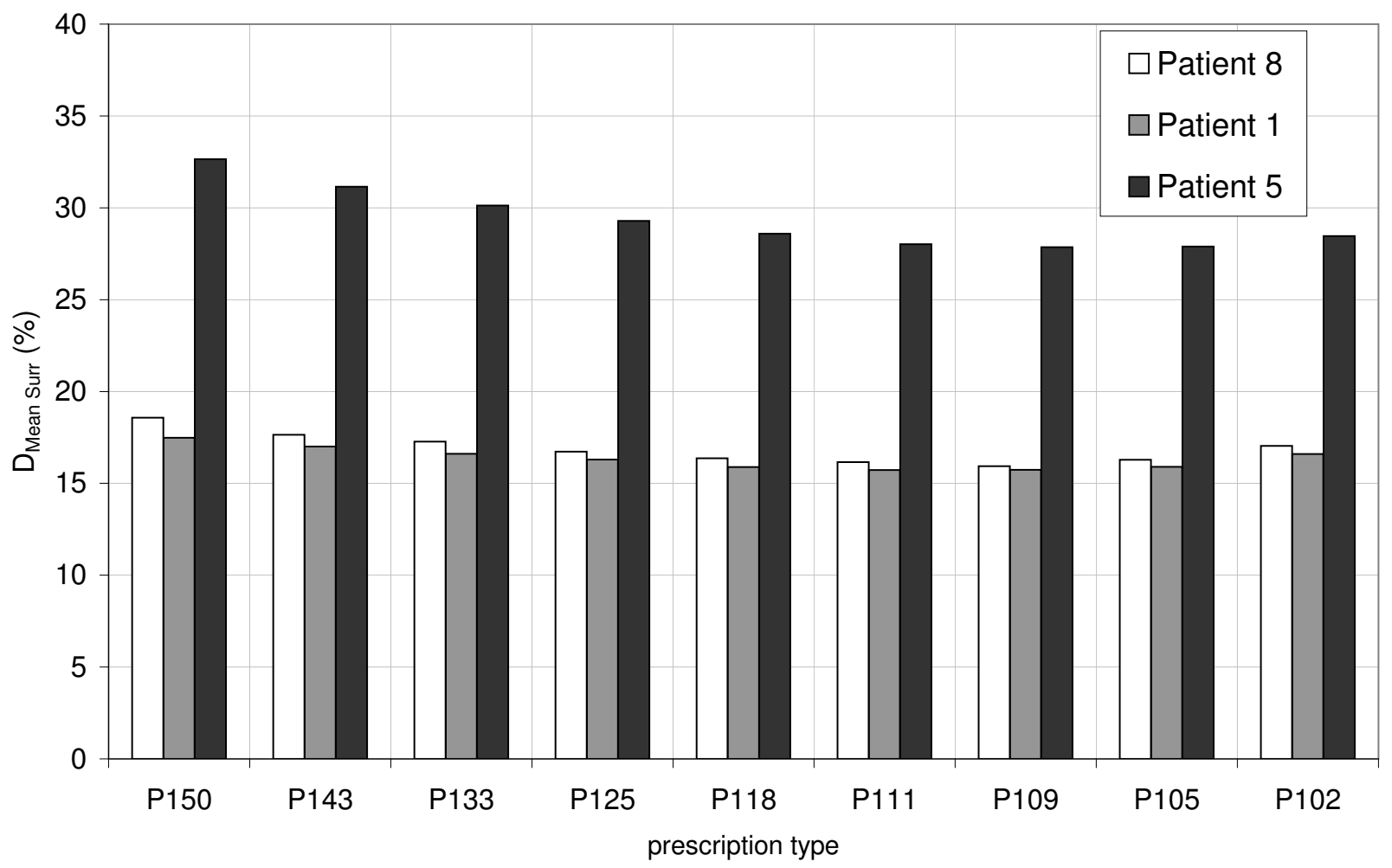

Figure 7

Accumulated mean dose to the surrounding area $D_{\text {Mean Surr }}$ as a function of the dose prescription for three patients (\#8, \#I, \#5).

\section{Discussion}

This study used a one-dimensional convolution model to simulate the effects of breathing induced target motion in radiotherapy treatment. We allowed dose inhomogeneity in the target, which is considered as the standard in SBRT treatment of intra-hepatic and intra-pulmonary tumors [29]. The dose inhomogeneity was achieved by changing field size and renormalization of the one-beam profile. No step-and-shoot segments were used to achieve dose inhomogeneity. Consequently, the results are of interest especially for conformal SBRT. However, the concept of inhomogeneous dose distributions for mobile target volumes may gain relevance for radiotherapy treatment in general.

The time-weighted mean tumor position was selected as the isocentre in this study. This set-up ensured, that minimum doses after consideration of breathing motion without addition of extra margins were identical at both "edges" of the target for nearly all patients. As a consequence, the addition of margins resulted in equal dose compensation to $100 \%$ at both edges of the target. A comparable concept with treatment planning based on the mean tumor position has been described by Wolthaus et al. [27]. Consequently, the time-weighted mean target position is considered as the appropriate reference for treatment planning of target volumes with clinically large breathing motion.

\section{Influence of PDF shape and target dose inhomogeneity on margin size}

The shape of the probability density function - i.e. how long does the target stay at which position - influenced margins for motion compensation. The correlation between safety margins and maximum range of tumor motion was suboptimal: different margins were calculated for different shapes of the tumor PDF despite identical motion range. The maximum motion range describes 


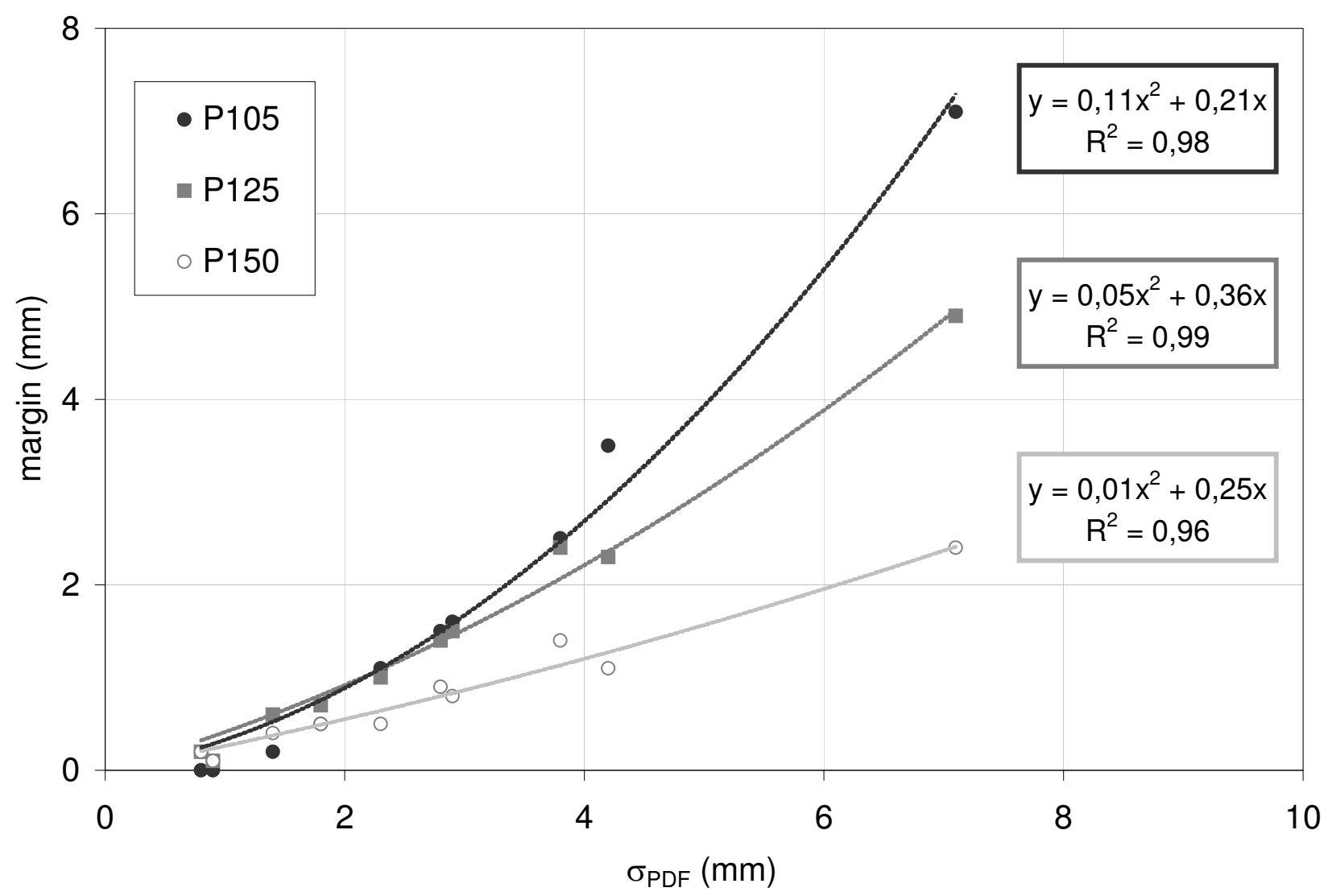

Figure 8

Margins for compensation of breathing induced dose loss to the target for all ten clinically observed patients.

the width of motion but not the shape of motion pattern. In all settings the standard deviation of the PDF was closely correlated to safety margins. This is in agreement to the findings of Jiang who showed the dose loss due to motion depends on the steepness of the dose profile and the shape of the PDF which is described by the standard deviation [30].

Table 2: Mean differences between internal target volume concept (ITV) and optimization of the surrounding isodose (OSI)

\begin{tabular}{cccc}
\hline & PI05 & PI25 & PI50 \\
\cline { 2 - 4 } & $3.6 \pm 0.7$ & $17.8 \pm 4.0$ & $37.1 \pm 8.9$ \\
$\Delta \mathbf{D}_{\text {Min }}$ in \% & $3.3 \pm 1.6$ & $3.6 \pm 2.3$ & $4.3 \pm 3.0$ \\
$\Delta$ Margin in mm & $7.8 \pm 3.0$ & $6.4 \pm 4.3$ & $4.9 \pm 3.1$ \\
$\Delta \mathbf{D}_{\text {Mean Surr in \% }}$ & & & \\
\hline
\end{tabular}

Abbreviations: absolute increase in minimum target dose $\Delta D_{\text {Min }}$, absolute increase in margin size $\Delta$ Margin, absolute increase in mean dose to surrounding region $\Delta D_{\text {Mean Surr }}$
We observed a quadratic relationship between margins for compensation of motion induced dose loss and the motion of the tumor expressed by the standard deviation. The quadratic relationship between safety margins and tumor motion was observed for all investigated degrees of dose inhomogeneity in the target volume but was most pronounced for P105 and least for P150, where a rather linear relationship between motion and margins was observed. A similar quadratic relationship has been described by Engelsman et al. and Sonke et al. [23,31]; these studies were limited to homogeneous dose distributions, which is the standard in conventionally fractionated radiotherapy.

The margins for compensation of breathing motion were smaller, if a higher degree of dose inhomogeneity was allowed in the target volume. Thus increased dose inhomogeneity reduced the absolute irradiated volume of the surrounding organ-at-risk. However, increased dose inhomogeneity in the target volume was associated with 
increased doses to the target surrounding organ-at-risk in the entrance and exit beam regions. These contrary effects of dose inhomogeneity showed an optimum with minimum mean dose to the surrounding organ-at-risk for dose prescriptions between P105 and P118. The risk of normal tissue injury should be minimal in this region of dose inhomogeneity with simultaneously identical minimum doses to the target.

Such a benefit of inhomogeneous dose distributions for treatment of pulmonary targets has been described by Engelsman et al. [32]: an iso-NTCP increase of the tumor control probability was observed for reduced field sizes with higher dose inhomogeneity in the target. In accordance to our study, an optimal dose inhomogeneity in the target volume was observed. Data from that study were based on phantom measurements with simulated, regular breathing motion. Results of our study were in agreement, and confirmed this concept for inhomogeneous dose distributions based on irregular breathing motion of real patient data.

The internal target volume concept is most frequently used in clinical practice: the amplitude of breathing induced target motion, target positions in end-inhalation and end-exhalation, define the size of target volume. The comparison of the ITV and OSI concept ascertained that the ITV concept over-estimated the influence of breathing motion [33] with unnecessary large margins. Increased doses to the normal tissue and higher as planned doses to the target are consequences.

\section{Limitations of the study}

Though convolution calculations are an accepted model for investigation of spatial and temporal uncertainties in radiotherapy, limitations of this method are well known $[17,18]$ and some issues need to be considered for correct interpretation of results from this study. We did not aim to give numerical data for motion compensation which can be directly transferred into clinical practice. The focus of our work was to show the relation between motion compensation and a potential benefit of dose inhomogeneity.

Tissue inhomogeneities, differences in density between the solid tumor and the surrounding normal tissue are not considered. This may be of minor relevance for SBRT treatment of intra-hepatic targets. However, for intra-pulmonary tumors a decreased dose deposit in the pulmonary tissue of lower density and in the periphery of the solid tumor is well known [34]. We have recently shown, that this effect significantly influences the 4D dose calculation for pulmonary targets [33]: motion of the tumor and of the surrounding normal tissue resulted in a variant, nonstatic dose distribution during the breathing cycle. Model- ling of such effects with a convolution model is not possible. Despite this limitation, we decided to perform this study with the convolution method, because this approach allowed systematic analysis of the parameters dose profile, field size, target size, motion range and shape of the PDF. Such a large number of simulations would not have been possible with realistic four-dimensional treatment planning.

The simulations in this study were limited to one spatial dimension. In all ten patients, breathing motion of the target was largest in superior-inferior direction and consequently, motion in this direction was simulated. However, breathing motion in other than superior-inferior direction is well known [1]. With predominantly co-planar beam set-up the dose gradient is usually less steep in anterior-posterior and left-right direction compared to superior-inferior. Subsequently, margins for compensation of motion in these directions are expected to be even smaller compared to the margins calculated in this study $[19,30,35]$.

The external breathing signal was used as surrogate for generation of the tumor PDF; the range of tumor motion was evaluated in respiratory correlated CTs. This method of PDF generation was chosen to obtain realistic tumor PDFs based on several breathing cycles. It is well known that the correlation between external surrogate and internal target motion is inconsistent $[8,10]$. However, internal and external motion is usually best correlated for target motion in superior-inferior direction, which was simulated in our study. Additionally, phase shifts between internal and external motion are truly relevant for 4D-CT reconstruction and gated radiotherapy, but such phase shifts would have no influence on the results of our study. Consequently, this procedure is not considered as relevant for interpretation of results of our study: the benefit of increased dose inhomogeneity in terms of reduced safety margins for motion compensation was observed for all patients with various shapes and ranges of the tumor trajectories.

The simulation was based on one respiratory correlated CT acquired for treatment planning. Inter-fractional changes of the patients' breathing motion and breathing pattern are not considered. However, several studies have shown that one single respiratory correlated planning CT study was representative for the duration for a SBRT and conventionally fractionated treatment course [36-38].

The PDF binning was set to 10 bins for each PDF which resulted in different PDF resolutions in space depending on the maximum motion amplitude. The choice was based on the fact, that in clinical routine always the same 
number $(8-10)$ of respiration phases is chosen for reconstruction of the $4 \mathrm{D}-\mathrm{CT}$.

\section{Clinical consequences}

Optimal sparing of the organ-at-risk surrounding the target was achieved for dose prescriptions P105 to P118. For higher dose prescriptions, the effect of smaller safety margins was overcompensated by increased doses in the entrance and exit regions of the radiation beams. The mean dose to the surrounding organ-at-risk was the basis for this conclusion. Recently however, several studies have shown that very small doses between 5 Gy and 13 Gy [3941] are most predictive for radiation induced pneumonitis. If this were true the pulmonary tissue in the entrance and exit regions of the radiation beams would be "sacrificed" regardless of the dose homogeneity in the target volume. In such a scenario, sparing of lung tissue in superior-inferior direction would be more clinically relevant with the consequence of a benefit for dose inhomogeneities greater than P125.

Two recent studies in the literature described four-dimensional treatment planning using respiratory correlated CT. These studies used the ITV target volume concept for SBRT of pulmonary [33] and liver tumors [42] and a comparison between dose inhomogeneity of P125 and P150 was performed in both studies. These studies confirmed that an increased dose inhomogeneity resulted in increased doses to the target without a significantly increased dose the surrounding organs-at-risk. These conclusions support the findings in our study: the concept of inhomogeneous dose distributions should be developed further in the future.

Margins in our study were calculated for compensation of intra-fractional uncertainties of the target position, only. Other uncertainties in the process of treatment planning and treatment delivery need to be compensated with extra margins. Uncertainties in image acquisition and contouring of mobile targets require additional margins. Even high-precision stereotactic patient positioning was shown to result in large errors of the target position especially because of base-line shifts of the tumor independently from the bony anatomy [37,38,43-45]: decreased tumor control probability and increased risk of damage to adjacent critical structures are consequences $[46,47]$. The effects of such set-up errors on the dose to the target are expected to be more pronounced for treatment planning with a significant dose inhomogeneity in the target volume and consequently steep dose gradients close to the tumor. Image-guidance has been effective for compensation of these inter-fractional uncertainties and is considered as prerequisite for such high-precision radiotherapy $[11,36,48]$.
It is essential, that such aggressive protocols with increased maximum doses need to be balanced with clinical, patient specific factors. Whereas high dose inhomogeneity might be clinically acceptable for targets in the periphery of the lung, one would be cautious for centrally located targets [49]. However, it is clear that the way of dose prescription gives an additional degree of freedom in treatment planning to the physician and the physicist.

\section{Conclusion}

It was demonstrated that the time-weighted mean tumor position and the standard deviation of breathing induced target motion are the most relevant parameters in radiotherapy treatment planning of mobile targets. A quadratic relationship between motion and margins for compensation of dose loss was observed for various degrees of dose inhomogeneity within the target volume. A margin reduction was possible by allowing an inhomogeneous dose distribution in the target. The internal target volume concept over-compensated breathing motion with the consequence of higher than planned doses to the target and increased dose to the lung.

\section{Competing interests}

The authors declare that they have no competing interests.

\section{Authors' contributions}

All authors read and approved the final manuscript. AR designed the study and the analysis, performed the simulations and revised the manuscript. KB, JW, TK and MF participated in the study design and revised the manuscript. JM participated in the study design and analysis and revised the manuscript. MG designed the study and participated in the study design and revised the manuscript.

\section{Acknowledgements}

We would like to thank Mark Gainey for proof reading of the manuscript.

\section{References}

I. Seppenwoolde Y, Shirato H, Kitamura K, Shimizu S, van Herk M, Lebesque JV, Miyasaka K: Precise and real-time measurement of 3D tumor motion in lung due to breathing and heartbeat, measured during radiotherapy. Int J Radiat Oncol Biol Phys 2002, 53:822-834

2. Underberg RW, Lagerwaard FJ, Cuijpers JP, Slotman BJ, van Sornsen de Koste JR, Senan S: Four-dimensional CT scans for treatment planning in stereotactic radiotherapy for stage I lung cancer. Int J Radiat Oncol Biol Phys 2004, 60:1283-1290.

3. Ford EC, Mageras GS, Yorke E, Rosenzweig KE, Wagman R, Ling CC: Evaluation of respiratory movement during gated radiotherapy using film and electronic portal imaging. Int J Radiat Oncol Biol Phys 2002, 52:522-53I.

4. Lagerwaard FJ, Van Sornsen de Koste JR, Nijssen-Visser MR, Schuchhard-Schipper RH, Oei SS, Munne A, Senan S: Multiple "slow" CT scans for incorporating lung tumor mobility in radiotherapy planning. Int J Radiat Oncol Biol Phys 2001, 5 I:932-937.

5. Meyer J, Richter A, Baier K, Wilbert J, Guckenberger M, Flentje M: Tracking Moving Objects With Megavoltage Portal Imaging: A Feasibility Study. Med Phys 2006, 33:. 
6. Alasti H, Cho YB, Vandermeer AD, Abbas A, Norrlinger B, Shubbar $S$, Bezjak $A$ : $A$ novel four-dimensional radiotherapy method for lung cancer: imaging, treatment planning and delivery. Phys Med Biol 2006, 5 I:325 I-3267.

7. Hanley J, Debois MM, Mah D, Mageras GS, Raben A, Rosenzweig K, Mychalczak B, Schwartz LH, Gloeggler PJ, Lutz W, et al.: Deep inspiration breath-hold technique for lung tumors: the potential value of target immobilization and reduced lung density in dose escalation. Int J Radiat Oncol Biol Phys 1999, 45:603-6II.

8. Keall PJ, Joshi S, Vedam SS, Siebers JV, Kini VR, Mohan R: Fourdimensional radiotherapy planning for DMLC-based respiratory motion tracking. Med Phys 2005, 32:942-95I.

9. Minohara S, Kanai T, Endo M, Noda K, Kanazawa M: Respiratory gated irradiation system for heavy-ion radiotherapy. Int J Radiat Oncol Biol Phys 2000, 47: 1097-I I03.

10. Rietzel E, Liu AK, Doppke KP, Wolfgang JA, Chen AB, Chen GT, Choi NC: Design of 4D treatment planning target volumes. Int J Radiat Oncol Biol Phys 2006, 66:287-295.

II. Shirato H, Suzuki K, Sharp GC, Fujita K, Onimaru R, Fujino M, Kato $\mathrm{N}$, Osaka Y, Kinoshita R, Taguchi H, et al.: Speed and amplitude of lung tumor motion precisely detected in four-dimensional setup and in real-time tumor-tracking radiotherapy. $\ln \mathrm{J}$ Radiat Oncol Biol Phys 2006, 64:1229-1236.

12. Wong JW, Sharpe MB, Jaffray DA, Kini VR, Robertson JM, Stromberg JS, Martinez AA: The use of active breathing control (ABC) to reduce margin for breathing motion. Int J Radiat Oncol Biol Phys 1999, 44:9|I-919.

13. Keall PJ, Mageras GS, Balter JM, Emery RS, Forster KM, Jiang SB, Kapatoes JM, Low DA, Murphy MJ, Murray BR, et al:: The management of respiratory motion in radiation oncology report of AAPM Task Group 76. Med Phys 2006, 33:3874-3900

14. Webb S: Motion effects in (intensity modulated) radiation therapy: a review. Phys Med Biol 2006, 5 I:R403-425.

15. Booth JT, Zavgorodni SF: Modelling the dosimetric consequences of organ motion at CT imaging on radiotherapy treatment planning. Phys Med Biol 200I, 46: I 369-I377.

16. Chetty IJ, Rosu M, Tyagi N, Marsh LH, McShan DL, Balter JM, Fraass BA, Ten Haken RK: A fluence convolution method to account for respiratory motion in three-dimensional dose calculations of the liver: a Monte Carlo study. Med Phys 2003, 30:1776-1780.

17. Craig T, Battista J, Van Dyk J: Limitations of a convolution method for modeling geometric uncertainties in radiation therapy. II. The effect of a finite number of fractions. Med Phys 2003, 30:2012-2020.

18. Craig T, Battista J, Van Dyk J: Limitations of a convolution method for modeling geometric uncertainties in radiation therapy. I. The effect of shift invariance. Med Phys 2003, 30:200I-20II.

19. Gordon JJ, Siebers JV: Convolution method and CTV-to-PTV margins for finite fractions and small systematic errors. Phys Med Biol 2007, 52: 1967-1990.

20. Leong J: Implementation of random positioning error in computerised radiation treatment planning systems as a result of fractionation. Phys Med Biol 1987, 32:327-334.

21. Lujan AE, Larsen EW, Balter JM, Ten Haken RK: A method for incorporating organ motion due to breathing into 3D dose calculations. Med Phys 1999, 26:715-720.

22. McCarter SD, Beckham WA: Evaluation of the validity of a convolution method for incorporating tumour movement and set-up variations into the radiotherapy treatment planning system. Phys Med Biol 2000, 45:923-931.

23. Engelsman M, Sharp GC, Bortfeld T, Onimaru R, Shirato H: How much margin reduction is possible through gating or breath hold? Phys Med Biol 2005, 50:477-490.

24. Guckenberger M, Weininger M, Wilbert J, Richter A, Baier K, Krieger $T$, Polat $B$, Flentje $M$ : Influence of retrospective sorting on image quality in respiratory correlated computed tomography. Radiother Oncol 2007.

25. Wulf J, Haedinger U, Oppitz U, Thiele W, Mueller G, Flentje M: Stereotactic radiotherapy for primary lung cancer and pulmonary metastases: a noninvasive treatment approach in medically inoperable patients. Int J Radiat Oncol Biol Phys 2004, 60:186-196.
26. Storchi PR, van Battum LJ, Woudstra E: Calculation of a pencil beam kernel from measured photon beam data. Phys Med Biol 1999, 44:2917-2928.

27. Wolthaus JW, Schneider C, Sonke J], van Herk M, Belderbos JS, Rossi MM, Lebesque JV, Damen EM: Mid-ventilation CT scan construction from four-dimensional respiration-correlated CT scans for radiotherapy planning of lung cancer patients. Int J Radiat Oncol Biol Phys 2006, 65:1560-1571.

28. Belderbos JS, Heemsbergen WD, De Jaeger K, Baas P, Lebesque JV: Final results of a Phase I/II dose escalation trial in non-smallcell lung cancer using three-dimensional conformal radiotherapy. Int J Radiat Oncol Biol Phys 2006, 66: I26-134.

29. Timmerman RD, Kavanagh BD, Cho LC, Papiez L, Xing L: Stereotactic body radiation therapy in multiple organ sites. J Clin Oncol 2007, 25:947-952.

30. Jiang R, Barnett RB, Chow JC, Chen JZ: The use of spatial dose gradients and probability density function to evaluate the effect of internal organ motion for prostate IMRT treatment planning. Phys Med Biol 2007, 52: I 469-1484.

31. Sonke J], Lebesque J, van Herk M: Variability of four-dimensional computed tomography patient models. Int J Radiat Oncol Biol Phys 2008, 70:590-598.

32. Engelsman $M$, Remeijer $P$, van Herk $M$, Lebesque JV, Mijnheer $B$, Damen EM: Field size reduction enables iso-NTCP escalation of tumor control probability for irradiation of lung tumors. Int J Radiat Oncol Biol Phys 200 I, 5 I: I 290-I 298.

33. Guckenberger M, Wilbert J, Krieger T, Richter A, Baier K, Meyer J, Flentje M: Four-dimensional treatment planning for stereotactic body radiotherapy. Int I Radiat Oncol Biol Phys 2007, 69:276-285

34. Haedinger U, Krieger T, Flentje M, Wulf J: Influence of calculation model on dose distribution in stereotactic radiotherapy for pulmonary targets. Int J Radiat Oncol Biol Phys 2005, 61:239-249.

35. van Herk M: Errors and margins in radiotherapy. Semin Radiat Oncol 2004, 14:52-64

36. Guckenberger M, Meyer J, Wilbert J, Baier K, Mueller G, Wulf J, Flentje M: Cone-beam CT based image-guidance for extracranial stereotactic radiotherapy of intrapulmonary tumors. Acta Oncol 2006, 45:897-906.

37. Guckenberger M, Wilbert J, Meyer J, Baier K, Richter A, Flentje M: Is a single respiratory correlated $4 \mathrm{D}-\mathrm{CT}$ study sufficient for evaluation of breathing motion? Int J Radiat Oncol Biol Phys 2007, 67:1352-1359.

38. Geld YG van der, Lagerwaard FJ, van Sornsen de Koste JR, Cuijpers JP, Slotman BJ, Senan S: Reproducibility of target volumes generated using uncoached 4-dimensional CT scans for peripheral lung cancer. Radiat Oncol 2006, I:43.

39. Schallenkamp JM, Miller RC, Brinkmann DH, Foote T, Garces YI: Incidence of radiation pneumonitis after thoracic irradiation: Dose-volume correlates. Int J Radiat Oncol Biol Phys 2007, 67:410-416.

40. Semenenko VA, Molthen RC, Li C, Morrow NV, Li R, Ghosh SN, Medhora MM, Li XA: Irradiation of varying volumes of rat lung to same mean lung dose: a little to a lot or a lot to a little? Int J Radiat Oncol Biol Phys 2008, 7 1:838-847.

4I. Wang S, Liao Z, Wei X, Liu HH, Tucker SL, Hu CS, Mohan R, Cox JD, Komaki R: Analysis of clinical and dosimetric factors associated with treatment-related pneumonitis (TRP) in patients with non-small-cell lung cancer (NSCLC) treated with concurrent chemotherapy and three-dimensional conformal radiotherapy (3D-CRT). Int J Radiat Oncol Biol Phys 2006, 66:1399-1407.

42. de Pooter JA, Wunderink W, Mendez Romero A, Storchi PR, Heijmen B]: PTV dose prescription strategies for SBRT of metastatic liver tumours. Radiother Oncol 2007, 85:260-266.

43. Bosmans G, van Baardwijk A, Dekker A, Ollers M, Boersma L, Minken A, Lambin P, De Ruysscher D: Intra-patient variability of tumor volume and tumor motion during conventionally fractionated radiotherapy for locally advanced non-small-cell lung cancer: a prospective clinical study. Int I Radiat Oncol Biol Phys 2006, 66:748-753.

44. Guckenberger M, Meyer J, Wilbert J, Richter A, Baier K, Mueller G, Flentje $M$ : Intra-fractional uncertainties in cone-beam $C T$ based image-guided radiotherapy (IGRT) of pulmonary tumors. Radiother Oncol 2007, 83:57-64. 
45. Uematsu M, Shioda A, Suda A, Tahara K, Kojima T, Hama Y, Kono M, Wong JR, Fukui T, Kusano S: Intrafractional tumor position stability during computed tomography (CT)-guided frameless stereotactic radiation therapy for lung or liver cancers with a fusion of CT and linear accelerator (FOCAL) unit. Int J Radiat Oncol Biol Phys 2000, 48:443-448.

46. Herfarth KK, Debus J, Lohr F, Bahner ML, Fritz P, Hoss A, Schlegel W, Wannenmacher MF: Extracranial stereotactic radiation therapy: set-up accuracy of patients treated for liver metastases. Int J Radiat Oncol Biol Phys 2000, 46:329-335.

47. Wulf J, Hadinger U, Oppitz U, Olshausen B, Flentje M: Stereotactic radiotherapy of extracranial targets: CT-simulation and accuracy of treatment in the stereotactic body frame. Radiother Oncol 2000, 57:225-236.

48. Purdie TG, Bissonnette JP, Franks K, Bezjak A, Payne D, Sie F, Sharpe $M B$, Jaffray DA: Cone-beam computed tomography for on-line image guidance of lung stereotactic radiotherapy: Localization, verification, and intrafraction tumor position. Int J Radiat Oncol Biol Phys 2007.

49. Timmerman R, McGarry R, Yiannoutsos C, Papiez L, Tudor K, DeLuca J, Ewing M, Abdulrahman R, DesRosiers C, Williams M, Fletcher J: Excessive toxicity when treating central tumors in a phase II study of stereotactic body radiation therapy for medically inoperable early-stage lung cancer. J Clin Oncol 2006, 24:4833-4839.

\section{Pre-publication history}

The pre-publication history for this paper can be accessed here:

http://www.biomedcentral.com/1756-6649/8/5/prepub

Publish with Bio Med Central and every scientist can read your work free of charge

"BioMed Central will be the most significant development for disseminating the results of biomedical research in our lifetime. "

Sir Paul Nurse, Cancer Research UK

Your research papers will be:

- available free of charge to the entire biomedical community

- peer reviewed and published immediately upon acceptance

- cited in PubMed and archived on PubMed Central

- yours - you keep the copyright

Submit your manuscript here:

http://www.biomedcentral.com/info/publishing_adv.asp 